\title{
Interference Spins: Scheduling of Multiple Interfering Two-Way Wireless Links
}

\author{
Petar Popovski, Senior Member, IEEE, Osvaldo Simeone, Senior Member, IEEE, \\ Jimmy J. Nielsen, Member, IEEE and Čedomir Stefanović, Member, IEEE
}

\begin{abstract}
Two-way is a dominant mode of communication in wireless systems. Departing from the tradition to optimize each transmission direction separately, recent work has demonstrated that, for time-division duplex (TDD) systems, optimizing the schedule of the two transmission directions depending on traffic load and interference condition leads to performance gains. In this letter, a general network of multiple interfering two-way links is studied under the assumption of a balanced load in the two directions for each link. Using the notion of interference spin, we introduce an algebraic framework for the optimization of twoway scheduling, along with an efficient optimization algorithm that is based on the pruning of a properly defined topology graph and dynamic programming. Numerical results demonstrate multi-fold rate gains with respect to baseline solutions, especially for worst-case $(5 \%$-ile) rates.
\end{abstract}

Index Terms-Two-way communication, scheduling, dynamic TDD, dynamic programming.

\section{INTRODUCTION}

Two-way is a dominant mode of communication in wireless systems, such as in uplink (UL)/downlink (DL) cellular communication. Although the two-way channel is the first known multi-user channel treated in information theory [1], the design of two-way links has traditionally been tackled by separating the problem into independent one-way DL and UL problems, respectively, that deal with rate adaptation, scheduling, etc.

Nevertheless, recent works [2]-[7] have demonstrated that the presence of interference motivates the joint consideration of UL and DL, since the interference caused by a link on other links is different depending on the direction in which the link is active, i.e., on which node acts as the transmitter. Specifically, the referenced works have studied dynamic Time Division Duplex (TDD) for small-cell wireless systems. With dynamic TDD, each slot, of possibly different size in the frequency-time plane, can be assigned to either the UL or DL direction, depending on traffic load and interference conditions. References [2] and [3] outline the challenge of managing UL-DL crossinterference in the presence of dynamic TDD. The works [4], [5] instead propose centralized and decentralized algorithms that optimize the switching time between UL and DL. Finally, [6], [7] put forth heuristic solutions for the optimization of UL-DL slot allocation for fixed switching times.

In this letter we consider a generic collection of two-way links, hence including, e.g., cellular and/or device-to-device

P. Popovski, J. J. Nielsen and Č. Stefanovicć are with the Department of Electronic Systems, Aalborg University, 9220 Aalborg, Denmark (e-mail: \{petarp, jjn, cs\}@es.aau.dk).

Osvaldo Simeone is with CWCSPR, New Jersey Institute of Technology, Newark, NJ 07102 USA (e-mail: osvaldo.simeone@njit.edu).

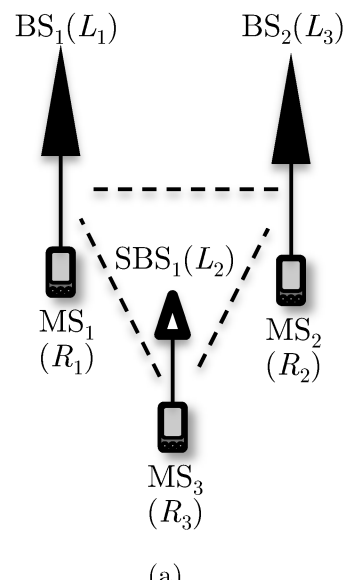

(a)

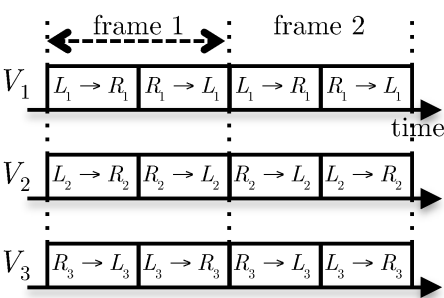

(b)

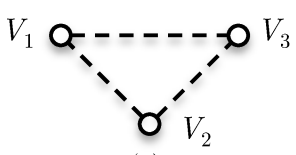

(c)
Fig. 1. (a) Example scenario with two macro-cells and one small cell, with direct channels (full lines) and interfering channels (dashed lines). (b) Example spin configuration in two subsequent frames. (c) Topology graph $\mathcal{G}$.

(D2D) links, where the traffic load is balanced between the two transmission directions: a slot assigned to one transmission direction is always followed by one allocated to the opposite direction. This assumption is per definition suitable for applications using interactive communication. An example setup is given in Fig. 1 (a), that consists of three interfering two-way links operating over two-slot frames, with one slot allocated to each direction as per Fig. 1 (b). We refer to the binary variable that determines the link direction in the first slot (the opposite to the one in the second slot) as the interference spin of a link (see precise definition in the next section). This allows us to propose an algebraic framework for the optimization of twoway scheduling, e.g., UL-DL slot allocation. The optimization consists of selecting the interference spins of all $M$ links in order to maximize a general sum-utility of the signal-to-noiseplus-interference ratios (SINRs) of the two-way links. The optimal solution has a complexity that scales as $2^{M}$. Besides the algebraic framework, our second contribution is a suboptimal, but efficient, algorithm that leverages the representation of the $M$ interfering two-way links as a graph of $M$ nodes. The proposed algorithm is based on pruning this topology graph according to a specific criterion and applying dynamic programming. The algorithm finds a spin configuration that is seen via numerical results to exhibit substantial performance gains over the baseline scheme in which the spins are not optimized. 


\section{System Model AND Problem Definition}

We consider a set $\mathcal{V}$ of $M$ synchronous two-way links, where the $l$-th link $V_{l}$ consists of two half-duplex nodes $L_{l}$ and $R_{l}$. The labeling of the two nodes in a link $V_{l}$ as left, $L_{l}$, and right, $R_{l}$, is arbitrary, see Fig. 1 Each link $V_{l}$ uses TDD, and two-way communication takes place in a frame consisting of two slots. The first slot is used in one direction, either $L_{l} \rightarrow R_{l}$ or $R_{l} \rightarrow L_{l}$, and the successive slot is used in the opposite direction. Related to this, we define interference spin or, for short, spin of a link in a given frame: the $l$-th link $V_{l}$ is said to have a left spin or $0-$ spin if $L_{l}$ transmits in the odd slot and $R_{l}$ transmits in the even slot; otherwise the link has a right spin or $1-$ spin in that frame. In the example in Fig. 1 b), the link $V_{2}$ has a left spin in the first frame and a right spin in the second frame. The spin of the $l$-th link is denoted by $s_{l} \in\{0,1\}$.

All devices use the same spectrum in a TDD manner, leading to interference among the concurrent link transmissions. Each device is backlogged with data, such that there is transmission in each slot and there is a continuous stream of slots. All links are slot-/frame-synchronous. Considering two links, say $V_{k}: L_{k}-R_{k}$ and $V_{l}: L_{l}-R_{l}$, we distinguish the following channel gains, see Fig. 2. (a) Direct channels: $L_{k} \rightarrow R_{k}, R_{k} \rightarrow L_{k}, L_{l} \rightarrow R_{l}$ and $R_{l} \rightarrow L_{l}$, whose signal-to-noise ratios (SNRs) are defined as $\mathrm{SNR}_{k}^{L R}, \mathrm{SNR}_{k}^{R L}$, $\mathrm{SNR}_{l}^{L R}$ and $\mathrm{SNR}_{l}^{R L}$, respectively; (b) Interfering channels: $L_{k} \rightarrow R_{l}, R_{k} \rightarrow L_{l}, L_{k} \rightarrow L_{l}$ and $R_{k} \rightarrow R_{l}$, whose interference-to-noise ratios (INRs) are defined as $\operatorname{INR}_{k l}^{L R}$, $\mathrm{INR}_{k l}^{R L}, \mathrm{INR}_{k l}^{L L}$ and $\mathrm{INR}_{k l}^{R R}$, respectively. The interference from $l$ to $k$, not depicted in Fig. 2] is represented in a similar manner. Even with perfect channel reciprocity, we generally have $\mathrm{SNR}_{k}^{L R} \neq \mathrm{SNR}_{k}^{R L}$ and $\mathrm{INR}_{k l}^{L R} \neq \mathrm{INR}_{l k}^{R L}$, since the nodes at the two ends of a link may use different powers. Some of these quantities can be zero: for instance, links may not interfere with each other, e.g. due to obstacles and yield $\mathrm{INR}_{l k}^{L R}=\mathrm{INR}_{k l}^{R L}=0$.

In order to capture the interference-related features of the network, we define the undirected topology graph $\mathcal{G}=(\mathcal{V}, \mathcal{E})$, where the set of vertices $\mathcal{V}$ represents the links $V_{l}$, as defined above, and an edge exists in the edge set $\mathcal{E}$ for a pair of links $V_{k}$ and $V_{l}$ in $\mathcal{L}$ if and only if at least one of the interfering powers $\mathrm{INR}_{k l}^{L R}, \mathrm{INR}_{k l}^{R L}, \mathrm{INR}_{k l}^{L L}, \mathrm{INR}_{k l}^{R R}, \mathrm{INR}_{l k}^{L R}$, $\mathrm{INR}_{l k}^{R L}, \mathrm{INR}_{l k}^{L L}$ and $\mathrm{INR}_{l k}^{R R}$ is non-zero. Therefore, an edge $k l \in \mathcal{E}$ indicates that two links $V_{k}$ and $V_{l}$ interfere at least in one direction. Note that we identify edges via the indices of the connected links. The topology graph for the example in Fig. 1(a) is depicted in Fig. 1(c). Without loss of generality, the graph is assumed to be connected [8], since, otherwise, one could consider the different connected components separately.

Denoting by $\oplus$ the XOR operation, the interference between two links $V_{k}$ and $V_{l}$ is fully specified by the relative spin $r_{k l}$ :

$$
r_{k l}=r_{l k}=s_{k} \oplus s_{l}
$$

The SINRs for any link $V_{l}$ in the two directions $L_{l} \rightarrow R_{l}$ and $R_{l} \rightarrow L_{l}$ can be written as a function solely of the relative spins of the interfering links, i.e., of $r_{k l}$ with $k l \in \mathcal{E}$.

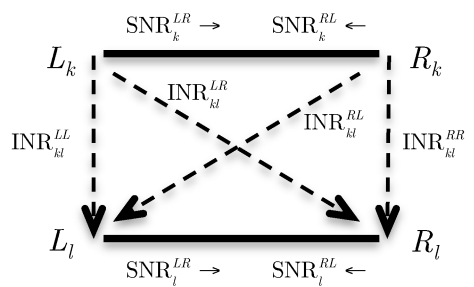

Fig. 2. Two interfering links $V_{k}: L_{k}-R_{k}$ and $V_{l}: L_{l}-R_{l}$. The INRs are given only for the interference from $V_{l}$ to $V_{k}$ for clarify of illustration.

Specifically, the SINR for the direction $L_{l} \rightarrow R_{l}$ is given as $\operatorname{SINR}_{l}^{L R}\left(\mathbf{r}_{l}\right)=\frac{\operatorname{SNR}_{l}^{L R}}{1+\sum_{k: k l \in \mathcal{E}}\left[\left(1-r_{k l}\right) \mathrm{INR}_{k l}^{L R}+r_{k l} \mathrm{INR}_{k l}^{R R}\right]}$

and for the direction $R_{l} \rightarrow L_{l}$ we have

$\operatorname{SINR}_{l}^{R L}\left(\mathbf{r}_{l}\right)=\frac{\mathrm{SNR}_{l}^{R L}}{1+\sum_{k: k l \in \mathcal{E}}\left[\left(1-r_{k l}\right) \mathrm{INR}_{k l}^{R L}+r_{k l} \mathrm{INR}_{k l}^{L L}\right]}$,

where $\mathbf{r}_{l}$ is the vector of spins $r_{k l}, k l \in \mathcal{E}$, for the link $V_{l}$. By rewriting the denominator of (2) as $1+\sum_{k: k l \in \mathcal{E}}\left[\operatorname{INR}_{k l}^{L R}+\right.$ $\left.r_{k l}\left(\operatorname{INR}_{k l}^{R R}-\mathrm{INR}_{k l}^{L R}\right)\right]$, we see that, if $\operatorname{INR}_{k l}^{R R}=\operatorname{INR}_{k l}^{L R}$, the relative spin $r_{k l}$ does not affect $\operatorname{SINR}_{l}^{L R}\left(\mathbf{r}_{l}\right)$. Similarly, if $\mathrm{INR}_{k l}^{L L}=\operatorname{INR}_{k l}^{R L}$, then $r_{k l}$ does not affect $\operatorname{SINR}_{l}^{R L}\left(\mathbf{r}_{l}\right)$.

Given a topology graph $\mathcal{G}$, definition (1) implies that the vector $\mathbf{r}=\left[\mathbf{r}_{1} \cdots \mathbf{r}_{M}\right]$ of all relative spins satisfies the following properties: (C1) Symmetry: $r_{k l}=r_{l k}$; (C2) Parity check on cycles: For any cycle in the topology graph $l_{1} l_{2}, l_{2} l_{3} \ldots, l_{N} l_{1} \in \mathcal{E}$, we have the parity check equation

$$
r_{l_{1} l_{2}} \oplus r_{l_{2} l_{3}} \oplus \ldots \oplus r_{l_{N-1} l_{N}} \oplus r_{l_{N} l_{1}}=0 .
$$

It can be easily shown that $\mathbf{C 1}$ and $\mathbf{C 2}$ are also sufficient to guarantee the existence of a vector of spins that satisfies (1) and hence they characterize the set of all relative spin vectors. A simple consequence of $\mathbf{C 1}$ and $\mathbf{C 2}$ is that the specification of the relative spins on the edges of any spanning tree [8] on the topology graph $\mathcal{G}$ is sufficient to obtain the entire vector $\mathbf{r}$. Every edge $k l$ either belongs to the tree and hence $r_{k l}$ is fixed, or, if not, then edge $k l$ forms a unique cycle with a subset of the edges of the tree and the relative spin follows from (4).

We are interested in finding the spin configuration that maximizes a sum-utility function of the SINRs across all links. The problem of interest is hence formulated as

$$
\max _{\mathbf{r}} \sum_{l=1}^{M} U_{l}\left(\operatorname{SINR}_{l}^{L R}\left(\mathbf{r}_{l}\right), \operatorname{SINR}_{l}^{R L}\left(\mathbf{r}_{l}\right)\right) \quad \text { s.t. } \mathbf{C 1} \text { and } \mathbf{C 2}
$$

where the maximization over the relative spins is subject to the constraints $\mathbf{C 1}$ and $\mathbf{C 2}$, and we have fixed non-negative utility functions $U_{l}(\cdot, \cdot)$ for each link $V_{l}$. The utility function $U_{l}(\cdot, \cdot)$ is assumed to be non-decreasing in the arguments and will be written as $U_{l}\left(\mathbf{r}_{l}\right)$ in order to simplify the notation. An example is the two-way sum-rate, which is given as $U_{l}\left(\mathbf{r}_{l}\right)=$ $\log \left(1+\operatorname{SINR}_{l}^{L R}\left(\mathbf{r}_{l}\right)\right)+\log \left(1+\operatorname{SINR}_{l}^{R L}\left(\mathbf{r}_{l}\right)\right)$. In principle, a link can change the spin per frame; however, in Sec. IV] we will treat the case in which a link spin is determined based on 
SINR variables that include long-term fading and update the the scheduling decisions on a large time scale.

\section{OPTIMIZING THE INTERFERENCE SPINS}

The maximization in (5), in principle, can be carried out by exploring all possible configurations of relative spins. Due to the constraint (4), the complexity of exhaustive search scales as $2^{M-1}$, becoming impractical as $M$ increases. Here we propose a suboptimal, but computationally more efficient optimization strategy that consists of two steps: 1) Construction of a maximum relative-interference spanning tree $\mathcal{T}$ over the graph $\mathcal{G}$; 2) Dynamic programming spin optimization on $\mathcal{T}$.

\section{1) Maximum Relative-Interference Spanning Tree $\mathcal{T}$}

For each edge $k l$ in $\mathcal{G}$, a weight $w_{k l}$ is assigned as

$$
\begin{array}{r}
w_{k l}=\max \left(\left|\mathrm{INR}_{k l}^{R R}-\mathrm{INR}_{k l}^{L R}\right|,\left|\mathrm{INR}_{k l}^{L L}-\mathrm{INR}_{k l}^{R L}\right|,\right. \\
\left.\left|\mathrm{INR}_{l k}^{R R}-\mathrm{INR}_{l k}^{L R}\right|,\left|\mathrm{INR}_{l k}^{L L}-\mathrm{INR}_{l k}^{R L}\right|\right) .
\end{array}
$$

This weight evaluates the maximum change in interference powers that is affected by the selection of the spin $r_{k l}$. A maximum spanning tree $\mathcal{T}$ is then constructed with respect to the weights in (6), retaining only the edges that have the largest contributions to the relative interference powers and pruning the remaining edges; e.g., for the example in Fig.1(c), one of the three edges will be removed based on the criterion (6), thus obtaining $\mathcal{T}$ with two edges. We refer to $\mathcal{T}$ as the maximum relative-interference spanning tree. The tree $\mathcal{T}$ can be constructed in a centralized or distributed way [8] [9], but the implementation details are out of the scope of this paper. Finally, a root vertex is arbitrarily selected so as to make $\mathcal{T}$ a rooted tree. Note that each vertex $V_{l}$ in $\mathcal{T}$ has a single parent vertex $P_{l}$ (except for the root vertex, which has no parent), i.e., $P_{l}$ is the only vertex in $\mathcal{T}$ along the unique path to the root. $V_{l}$ can be the parent for multiple children vertices $\mathcal{C}_{l}$.

\section{2) Dynamic Programming}

Having constructed the spanning tree $\mathcal{T}$, we now proceed to optimize only the relative spins corresponding to the edges $k l \in \mathcal{T}$ via dynamic programming. As discussed in Sec. the spins for all the remaining edges can then be immediately calculated via (4). In order to allow the optimization to be limited only to the edges in $\mathcal{T}$, we approximate the SINRs in (2) and (3) so that they only depend on the relative spins for the edges in $\mathcal{T}$. To this end, denote by $\mathbf{r}_{\mathcal{C}_{l} l}$ the vector of the relative spins for the edges connecting the child nodes $\mathcal{C}_{l}$ with $V_{l}$ and, similarly, by $r_{l P_{l}}$ the relative spin between $V_{l}$ and its parent $P_{l}$. The SINR (2) for link $V_{l}$ is approximated as

$$
\begin{aligned}
& \operatorname{SINR}_{l}^{L R}\left(\mathbf{r}_{\mathcal{C}_{l} l}, r_{l P_{l}}\right)=\frac{\mathrm{SNR}_{l}^{L R}}{1+\sum_{k: k l \in \mathcal{E}} \mathrm{INR}_{k l}}, \text { where } \\
& \operatorname{INR}_{k l}= \begin{cases}\left(1-r_{k l}\right) \mathrm{INR}_{k l}^{L R}+r_{k l} \mathrm{INR}_{k l}^{R R} & k \in \mathcal{C}_{l} \cup\left\{P_{l}\right\}, \\
\operatorname{INR}_{k l}^{L R}+\left(\mathrm{INR}_{k l}^{L R}+\mathrm{INR}_{k l}^{R R}\right) / 2 & \text { else. }\end{cases}
\end{aligned}
$$

In other words, the interference contribution for the edges $k l \in \mathcal{E} \backslash \mathcal{T}$ that do not belong to the tree is approximated with the average of the interference powers that would be observed if $r_{k l}=0$ or $r_{k l}=1$. This is justified by the fact that, in light of the choice of the weights (6), it is expected that the two values $\operatorname{INR}_{k l}^{L R}$ and $\operatorname{INR}_{k l}^{R R}$ are similar. The same approach is used to approximate (3), leading to the approximation $\operatorname{SINR}_{l}^{R L}\left(\mathbf{r}_{\mathcal{C}_{l} l}, r_{l P_{l}}\right)$. For ease of notation, we write $\hat{U}_{l}\left(\mathbf{r}_{\mathcal{C}_{l} l}, r_{l P_{l}}\right)=U_{l}\left(\operatorname{SINR}_{l}^{L R}\left(\mathbf{r}_{\mathcal{C}_{l} l}, r_{l P_{l}}\right), \operatorname{SINR}_{l}^{R L}\left(\mathbf{r}_{\mathcal{C}_{l} l}, r_{l P_{l}}\right)\right)$.

We now aim at optimizing problem (5) with utilities $\hat{U}_{l}$ in lieu of $U_{l}$ for all $l \in \mathcal{V}$. The proposed dynamic programming solution starts from the leaf vertices and proceeds according to the (partial) order defined by the tree until the root is reached. In particular, each vertex $V_{l}$ calculates the message $\boldsymbol{\mu}_{l}=\left(\mu_{l}^{0}, \mu_{l}^{1}\right)$ for its parent vertex $P_{l}$, where

$$
\mu_{l}^{i}=\max _{\mathbf{r}_{\mathcal{C}_{l} l}}\left(\hat{U}_{l}\left(\mathbf{r}_{\mathcal{C}_{l} l}, r_{l P_{l}}=i\right)+\sum_{k \in \mathcal{C}_{l}} \mu_{k}^{r_{k l}}\right),
$$

for $i=0,1$. The maximization in (9) is over the relative spins corresponding to edges stemming from the child vertices $\mathcal{C}_{l}$. We denote a solution of the problem (9) as $\overline{\mathbf{r}}_{\mathcal{C}_{l} l}^{i}$ for $i=0,1$. Note that the leaves have no child vertices, and hence, for every leaf $V_{l}$, the message is calculated as $\mu_{l}^{i}=\hat{U}_{l}\left(r_{l P_{l}}=i\right)$ for $i=0,1$. Instead, the root vertex $t$, which has no parent, solves the problem $\max _{\mathbf{r}_{\mathcal{C}_{t} t}}\left(\hat{U}_{t}\left(\mathbf{r}_{\mathcal{C}_{t} t}\right)+\sum_{k \in \mathcal{C}_{t}} \mu_{k}^{r_{k t}}\right)$ and obtains an optimal solution $\overline{\mathbf{r}}_{\mathcal{C}_{t} t}$. A complete solution $\overline{\mathbf{r}}$ is finally obtained by backpropagation: starting with the children of the root vertex, each child vertex $k \in \mathcal{C}_{l}$ of a vertex $V_{l}$ selects the solution $\overline{\mathbf{r}}_{\mathcal{C}_{l} l}^{\bar{r}_{k l}}$, until the process reaches the leaves.

The complexity of the maximum spanning tree construction scales as $G \log M$ [8] [9], where $G \leq M(M-1) / 2$ and $M$ are the number of edges and vertices in $\mathcal{G}$, respectively. The order of complexity of the relative-spin optimization is $M \cdot 2^{D}$, where $D$ is the maximum number of children of a vertex in $\mathcal{T}$. While in the worst case, $D=M-1$, for a typical topology graph $\mathcal{G}$, we found $D$ to be much smaller than $M$, leading to significant complexity saving with respect to exhaustive search, see Section IV

\section{NUMERICAL RESUltS AND CONCLUDING REMARKS}

For performance evaluation, a small-cell set-up is considered over a $100 \mathrm{~m} \times 100 \mathrm{~m}$ area. $M$ links, or equivalently $2 M$ nodes, are generated as follows: $M$ transceivers are placed uniformly in this area and chosen equiprobably to be either an $L-$ or $R$ - node; then, the opposite node of the link ( $R$ or $L$, respectively) is placed at a uniformly selected random angle with distance $d$. Note that the opposite node may lie outside the area at hand. Two types of links are considered: (i) symmetric D2D links, with $d=d_{s}=10 \mathrm{~m}$; (ii) asymmetric femtocell links with $d=d_{a}=50 \mathrm{~m}$. These links differ as explained below. The long-term SNRs and INRs that are used by the algorithm to determine the configuration of relative spins account only for large-scale fading and path loss. The long-term SNR parameters for a symmetric link $V_{l}$ are given as $\mathrm{SNR}_{l}^{R L}=\mathrm{SNR}_{l}^{L R}=\mathrm{SNR}_{s} \beta_{l}$. The transmit power is only adapted to the path loss in order to get $\mathrm{SNR}_{s}=20 \mathrm{~dB}$, while $\beta_{l}$ represent independent log-normal shadowing with standard deviation $8 \mathrm{~dB}$. For an asymmetric link $V_{l}$, the $\mathrm{SNRs}$ are given as $\mathrm{SNR}_{l}^{L R}=\mathrm{SNR}_{a}^{L R} \beta_{l}$ and $\mathrm{SNR}_{l}^{R L}=\mathrm{SNR}_{a}^{R L} \beta_{l}$ with 


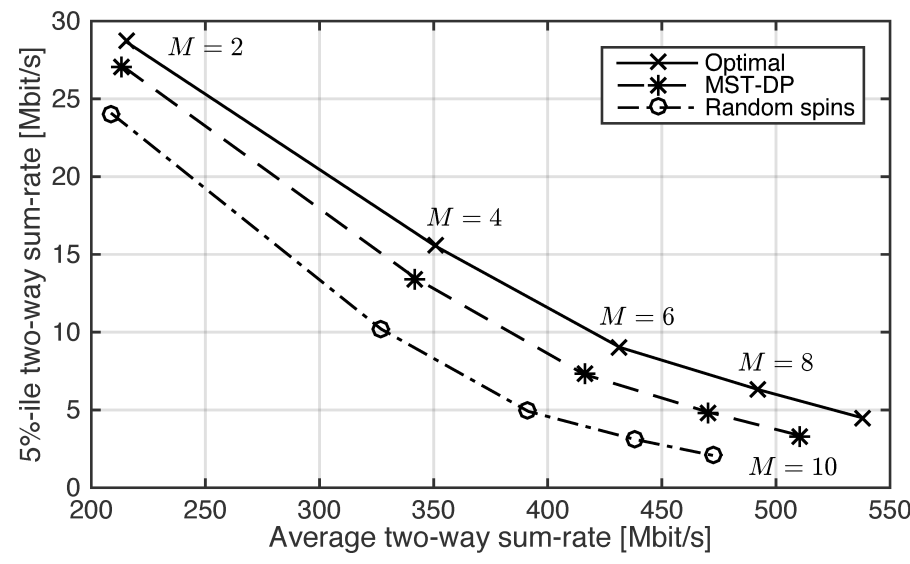

Fig. 3. 5\%-ile two-way sum-rate (for a bandwidth of $10 \mathrm{MHz}$ ) versus the average two-way sum-rate for different number of links $M$.

$\mathrm{SNR}_{a}^{L R}=20 \mathrm{~dB}$ and $\mathrm{SNR}_{a}^{R L}=10 \mathrm{~dB}$ due to the fact that the power of the femto-base station $(L)$ is different from the power of the mobile user $(R)$. The long-term interference power caused by the node $X_{l}$ to the node $Y_{k}$, where $X, Y \in\{L, R\}$, is given as $\operatorname{INR}_{l k}^{X Y}=\operatorname{SNR}_{z}^{X \bar{X}}\left(d_{z} / d_{l k}^{X Y}\right)^{\eta} \beta_{l k}^{X Y}$, where $z=s$ if $V_{l}$ is symmetric and $z=a$ otherwise, $X \bar{X} \in\{L R, R L\}$, $\eta=4$ is the path loss exponent, $d_{l k}^{X Y}$ is the distance between node $X_{l}$ and $Y_{k}$, and $\beta_{l k}^{X Y}$ accounts for log-normal shadowing with standard deviation $8 \mathrm{~dB}$. Note that $\beta_{l k}^{X Y}=\beta_{k l}^{Y X}$.

For a fixed spatial configuration and large-scale fading, the spins are optimized with the proportional fairness utility across the links, so that $U_{l}\left(\mathbf{r}_{l}\right)=\log \left(\log \left(1+\operatorname{SINR}_{l}^{L R}\left(\mathbf{r}_{l}\right)\right)+\log (1+\right.$ $\left.\left.\operatorname{SINR}_{l}^{R L}\left(\mathbf{r}_{l}\right)\right)\right)$. The proposed algorithm is referred to as MSTDP (Maximum Spanning Tree-Dynamic Programming). One reference, when computationally feasible, is the the optimal performance based on exhaustive search; the other is the average performance with uniform random spins. For a fixed spatial configuration, large-scale fading and relative spins, we evaluate for each link $V_{l}$ the instantaneous two-way sumrate $\log \left(1+\operatorname{sinr}_{l}^{L R}\left(\mathbf{r}_{l}\right)\right)+\log \left(1+\operatorname{sinr}_{l}^{R L}\left(\mathbf{r}_{l}\right)\right)$, where the instantaneous SINRs $\operatorname{sinr}_{l}^{L R}\left(\mathbf{r}_{l}\right)$ and $\operatorname{sinr}_{l}^{R L}\left(\mathbf{r}_{l}\right)$ are calculated via (2) and (3) by multiplying the corresponding long-term SNR and INR variables by unit-power Rayleigh variables, independently drawn for each par of nodes in each frame.

In Fig. 3, we plot the 5\%-ile of the two-way sum-rate, which is a common measure of worst-case rate, against the average two-way sum-rate, for different number $M$ of symmetric links and a bandwidth of $10 \mathrm{MHz}$. Note that having more links $M$ enables the spatial reuse, and hence the sumrate, to be improved, but, at the same time, it creates more interference that reduces the worst-case rates. It is seen that spin optimization can significantly outperform random-spin assignment, especially in terms of 5\%-ile rate, which, as an example, is nearly doubled for $M=10$.

Fig. 4 elaborates further on the 5\%-ile performance gains attained by optimized spin assignment with respect to randomspin selection as a function of $M$. Note that the high number of links is justified by the ultra-dense wireless scenarios envisioned in the upcoming $5 \mathrm{G}$ wireless systems [7]. Two curves are shown, one for all symmetric and one for all

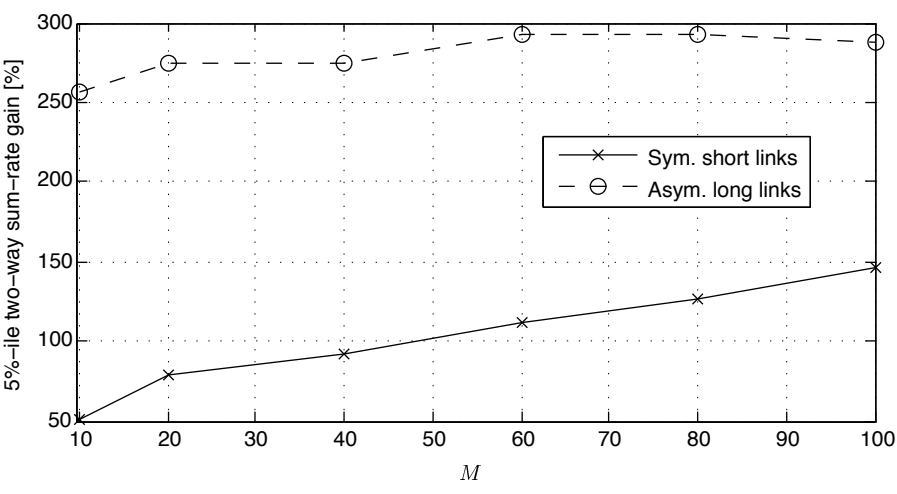

Fig. 4. Gain in 5\%-ile two-way sum-rate for the proposed MST-DP algorithm with respect to a random spin selection versus the number of links.

asymmetric links, respectively. For computational feasibility, only the MST-DP algorithm is considered. It is seen that, while the performance gains are substantial in both scenarios, optimizing the interference spins is particularly advantageous for asymmetric links. This is expected, since controlling the interference caused by the nodes that transmit with larger power has a more pronounced impact on the worst-case twoway sum-rate. Finally, the MST-DP algorithm, while inferior to the exhaustive search, still provides significant gains over random spins. We observe that, in terms of complexity, even with $M=100$ nodes, the value of $D$ was found to be always $D \leq 9$ and to be, on average less than 6 .

The main conclusion is that the degree of freedom offered by the interference spins leads to remarkable throughput gains, especially for the worst-case $(5 \%$-ile) rates.

\section{REFERENCES}

[1] C. E. Shannon, "Two-way communication channels," in Proc. 4th Berkeley Symp. Math. Stat. Prob.,, vol. 1, Berkeley, CA, 1961, pp. 611-644.

[2] B. Yu, S. Mukherjee, H. Ishii, and L. Yang, "Dynamic TDD support in the LTE-B enhanced Local Area Architecture," in Proc. IEEE Globecom 2012, Anaheim, CA, USA, Dec. 2012.

[3] Z. Shen, A. Khoryaev, E. Eriksson, and X. Pan, "Dynamic uplinkdownlink configuration and interference management in TD-LTE," IEEE Commun. Mag., vol. 50, no. 11, pp. 51-59, Nov. 2012.

[4] A. Dowhuszko et al, "A decentralized cooperative Uplink/Downlink adaptation scheme for TDD Small Cell Networks," in Proc. IEEE PIMRC 2013, London, UK, Sept. 2013.

[5] M. S. El Bamby, M. Bennis, W. Saad and M. Latva-Aho,"Dynamic Uplink-Downlink optimization in TDD-based small cell networks," in Proc. IEEE INFOCOM 2014, Toronto, Canada, Apr. 2014.

[6] D. Zhu and M. Lei, "Cluster-based dynamic DL/UL reconfiguration method in centralized RAN TDD with trellis exploration algorithm," in Proc. IEEE WCNC 2013, Shanghai, China, Apr. 2013.

[7] V. Venkatasubramanian, M. Hesse, P. Marsch and M. Maternia, "On the performance gain of flexible UL/DL TDD with centralized and decentralized resource allocation in dense 5G deployments," in Proc. IEEE PIMRC 2014, Washington, DC, USA, Sept. 2014.

[8] T. H. Cormen, C. E. Leiserson, R. L. Rivest, and C. Stein, Introduction to Algorithms, 2nd Ed. MIT Press and McGraw-Hill, 2001.

[9] R. G. Gallager, P. A. Humblet, and P. M. Spira, "A distributed algorithm for minimum-weight spanning trees," ACM Trans. Program. Lang. Sys., vol. 5, no. 1, pp. 66-77, Jan. 1983. 